\title{
Management of Radial Nerve Palsy Associated with Humeral Shaft Fractures by Closed Interlocking Intrameduallary Nail
}

\section{Mohamed Mansour Elzohairy*}

Lecturer and consultant of Orthopaedic Surgery, Faculty of Medicine, Zagazig University, Egypt

Clinical orthopaedic fellow, Toronto University, Canada

\begin{abstract}
Purpose: The purpose of this prospective study was to assess the results of immediate radial nerve palsy associated with closed humeral shaft fractures treated by the closed interlocking intramedullary nail without radial nerve exploration.

Methods: Eighteen patients suffered from immediate radial nerve palsy associated with closed humeral shaft fractures treated by closed interlocking intramedullary nail, without exploration of the radial nerve.

Results: Patients were followed up for a mean of 18 months (range from 12 to 24 months). All the cases had united in a good alignment within 12 weeks. Full clinical and electrodiagnostic radial nerve recovery as regarding the range of shoulder, elbow, wrist and hand movements occurred at 6 months examination.

Conclusion: Closed interlocking nailing for immediate radial nerve palsy associated with closed humeral shaft fractures, provides immediate stability and can be done with a closed technique, brief operative time and minimum violation to the radial nerve, with early return of optimal extremity function.
\end{abstract}

Keywords: Radial nerve; Palsy; Humeral shaft fractures; Interlocking intrameduallary nail

\section{Introduction}

Radial nerve palsy, either axonotmesis or neurapraxia, associated with fractures of the shaft humerus occurred in about 10 percent of the patients with humeral shaft fractures $[1,2,3]$. Its close relation to the humerus, especially fractures of the middle and the distal thirds of the humerus makes the radial nerve vulnerable to such injuries $[4,5,6]$. There are many debate about the management of radial nerve palsy associated with humeral shaft fractures especially the need for operative exploration [1]. The purpose of this study was to evaluate the results of eighteen patients suffered from immediate radial nerve palsy (occurring at the time of injury), associated with humeral shaft fractures treated by closed interlocking intramedullary nail. All the patients gave the informed consent prior to being included into this study; the study was authorized by the local ethical committee and was performed in accordance with the ethical standards of the 1964 Declaration of Helsinki as revised in 2000.

\section{Material and Methods}

Eighteen patients treated at Zagazig University Trauma Hospital, Sharkia, Egypt, (which considered as a big referral center for this type of cases and serves about twenty millions populations) from immediate radial nerve palsy associated with closed humeral shaft fractures treated by closed interlocking intramedullary nail. They were ten males and eight females their ages varying from 19 to 45 years (mean 32 years). Seven of them were associated with another fractures (multiple trauma patients) while eleven were single humeral shaft fractures. As regarding the inclusion criteria, we selected the cases with humeral shaft fractures in which the fracture sites varying from the middle third and the junction between the middle and the lower third (the site of the humerus makes the nerve vulnerable to injury). The fracture pattern were, one segmental, two comminuted, eight transverse and three short oblique and four long oblique. The causes of trauma were varying from car accidents in eight patients, motorcycle in six patients and fall from heights in four patients. The cases with compound and secondary radial nerve palsy were excluded from our study.

\section{Operative technique}

General anesthesia with intubation was used in all cases. Antegrade closed interlocking humeral nails procedure for the fixation of humeral shaft fractures, without radial nerve exploration was done for all the cases. Supine or beach chair positions with radiolucent side arm fixed to the radiolucent table were used for the patients. Image intensifier was used in all cases, a deltoid-splitting approach was used, the incision was started at the anterior aspect of the acromioclavicular joint and extended about $2.5 \mathrm{~cm}$ to $3 \mathrm{~cm}$. The site of entry for the nail into the humerus was in the sulcus between the greater tuberosity and the articular surface of the humeral head, directly in line with the medullray canal. We ream the intramedullary space beyond the fracture site over the guide wire with gradual increase in the reamer sizes till we reach about $1 \mathrm{~mm}$ above the nail chosen size, by this method we decreased the incidence of impaction of the nail or distraction at the facture site and decreased the risk of damaging radial nerve. Then the nail advanced over a guide wire, (Figure 1A and 1B). Two interlocking screws were used for proximal locking from the lateral side and one or two interlocking screws from anterior to posterior for distal locking according to the stability of distal fragment.Wounds were closed in layers, no suction drain used. Sutures were removed after two weeks later.

*Corresponding author: Mohamed Mansour Elzohairy, Lecturer and consultant of Orthopaedic Surgery, Faculty of Medicine, Mansour Elzohairy villa, 4 Zeid Ben Sabet Street, University villas, Zagazig, Sharkia Governorate, Egypt, Tel: +201124467856; E-mail: elzohairy2020@yahoo.com

Received March 03, 2012; Accepted March 22, 2012; Published March 25, 2012

Citation: Elzohairy MM (2012) Management of Radial Nerve Palsy Associated with Humeral Shaft Fractures by Closed Interlocking Intrameduallary Nail. J Trauma Treatment 1:121. doi:10.4172/2167-1222.1000121

Copyright: ( 2012 Elzohairy MM. This is an open-access article distributed unde the terms of the Creative Commons Attribution License, which permits unrestricted use, distribution, and reproduction in any medium, provided the original author and source are credited. 

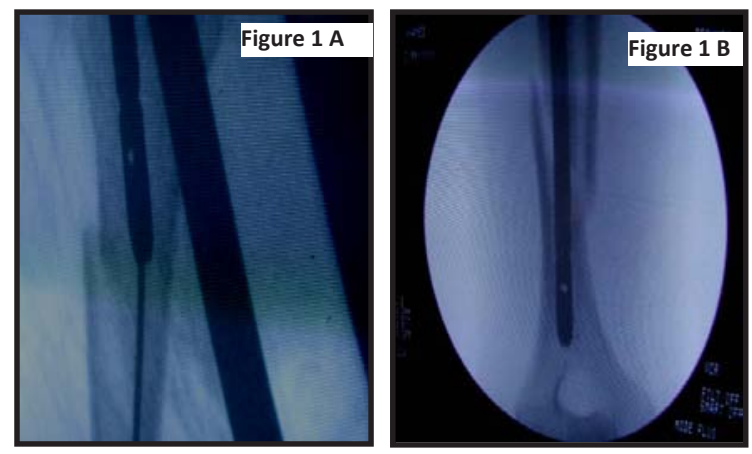

Figures 1: (A, B) The nail advanced over a guide wire under Fluoroscopic guidance.

\section{Post operative care}

Cock up splint was used for all the cases and physiotherapy started from the second postoperative day. Radial nerve electrodiagnostic studies were done at 2 weeks then at 6 weeks, 12 weeks, 4 months and 6 months for all the cases.

\section{Results}

The patients were followed up from 12 to 24 months (mean of 18 months). In all the cases complete union in a good alignment (no malalignment) of the fractures were achieved within 12 weeks (Figure 2A and 2B). All the wounds were healed with clean small scar (Figure 3). As regarding the electrodiagnostic nerve studies done in fifteen patients were as following: the EMG there was no denervation fibrillation potentials and the nerve conduction study showed delayed response (low amplitude) which coincides with neurapraxia as Seddon classification or first degree as Sunderland classification. Then the nerve conduction improved gradually with subsequent examinations. The electrodiagnostic nerve studies done in three patients were as following: the EMG there were denervation fibrillation potentials and the nerve conduction showed delayed response (low amplitude) followed by nascent potentials (highly polyphasic motor unit action potentials with low amplitude and long duration) which coincides with axontmesis as Seddon classification or second degree as Sunderland classification. Then the EMG and the nerve conduction study improved gradually with subsequent examinations. The clinical examination and electrodiagnostic nerve studies showed full radial nerve recovery with normal range of motion of shoulder, elbow, wrist and hand movements occurred at 6 months examination in all cases (Figure 4A,4B,4C and 4D). The ranges were as following : Shoulder motion (adduction /abduction 45-170 degrees, external rotation/ internal rotation with (shoulder abduction 90 degrees during test of shoulder rotation) the ranges were 90-80 degrees, flexion 165 degrees, extension 45 degrees), elbow motion were ( extension/ flexion 0-140 degrees, supination/ pronation 90-90 degrees) wrist and hand movements (dorsiflexion 70 degrees, palmarflexion 80 degrees, radial deviation 20 degrees, ulnar deviation 30 degrees), fingers flexion and extension at the metacarpophalangeal joints (flexion 90 degrees, extension 30-45 degrees), fingers adduction/ abduction 0-20 degrees), Thumb adduction/abduction 0-70 degrees) with normal fingers opposition.

NB (considering every movement started from zero. The 170 degrees shoulder abduction represents the two movements of glenohumeral and the scapulothoracic joints). Three cases $(14.3 \%$ incidence) were suffered from recurrent postoperative shoulder pain two years after the operation at the site of the nail entery especially after prolonged exhaustion of the shoulder which relieved by physiotherapy and analgesics.

\section{Discussion}

Conflicting views had been raised as regarding the need for operative exploration of the radial nerve in the cases of humeral shaft fractures associated with radial nerve palsy [1,7]. Jinn [8] in his series treated 162 humeral shaft fractures, 12 of them suffered from radial nerve palsy; he stated that the radial nerve exploration was mandatory for the cases with external rotation spiral humeral shaft fractures due to high incidence of radial nerve entrapment in such type of fractures. On the other hand, Pollock et al. [9] in their series reviewed 23 patients, with radial nerve palsy associated with fracture humerus who managed by closed method. They found that complete spontaneous recovery occurred in all (96\%) except one, due to nerve entrapment in the callus, who needed exploration with neurolysis after 14 weeks. Similarly, Sonneveled et al. [10], Böstman et al. [11,12] and Ring et al. [13] in their studies concluded that, routine early exploration of radial nerve in the cases suffered from radial nerve palsy associated with fracture humerus are not obligatory and not supported. Shao et al. [7] in their series of 11 patients had Holstein-Lewis [14] fractures of the humeral shaft, and in their review of English and German papers published from 1964 to 2005 that included at least 10 patients with radial nerve palsy associated with humeral shaft fractures they disagree with the concept of Holstein-Lewis [14] that, a spiral fracture pattern of the distal humerus with associated radial nerve palsy is an absolute indication for the radial nerve exploration. Also Shah et al. [15] concluded in their study the same opinion. Electromyography is valuable to demonstrate residual innervations or retained motor unit potentials during attempted volitional contraction that could be so minimal as to be undetected clinically. As wallerian degeneration
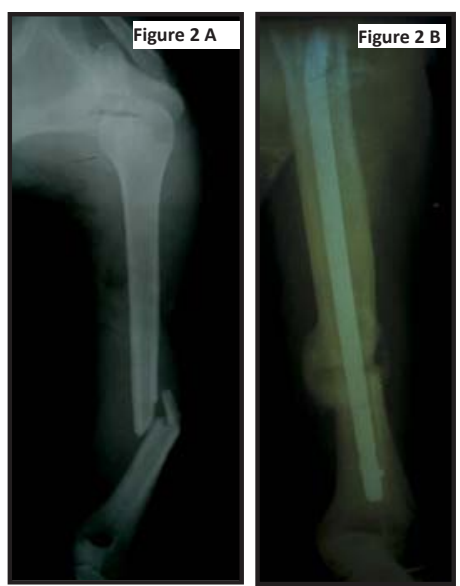

Figures 2: (A) Preoperative $X$ ray showed comminuted fracture humerus of 20-year-old female (B) 6 months fellow up showed the fracture with complete union and callus formation.

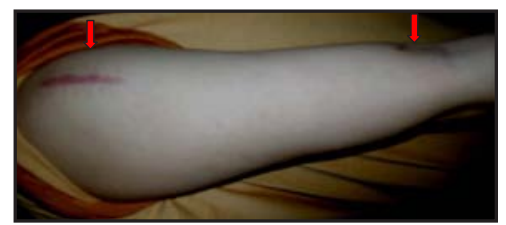

Figure 3: The postoperative small scar. 

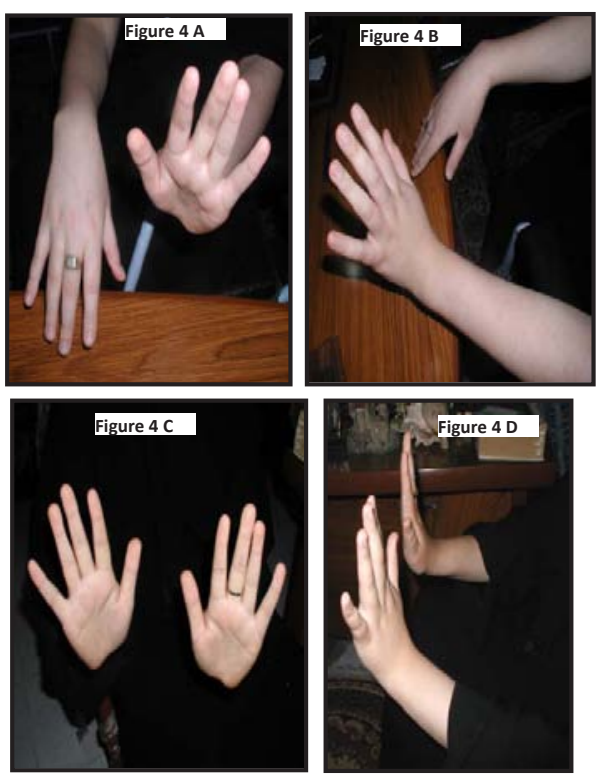

Figures 4: $(A, B)$ wrist drop 2 weeks clinical examination after injury $(C, D)$ full clinical radial nerve recovery occurred at 6 months examination.

progresses, fibrillation may be detected on electrode insertion at 7 to 14 days; 15 to 30 days after complete interruption of its motor supply, spontaneous fibrillation potentials of the muscle will be evident. If denervation fibrillation potentials have not appeared by the end of the second week, this may be considered a good prognostic sign. Fibrillations of denervation will last indefinitely until the muscle has become either reinnervated or fibrotic. Denervation potentials may increase transiently and then progressively decrease as reinnervation progresses. Evidence of reinnervation is judged to be present when highly polyphasic motor unit potentials are detected on attempts at volitional activity. Initially these may be of low amplitude with diphasic or triphasic configurations of short duration. With progression of reinnervation, these become numerous and of higher amplitude and eventually become more normal in configurations. By the same token, reinnervation potentials may be evident after regeneration of only a few motor fibres, and thus the presence of reinnervation potentials does not necessarily indicate that good return of volitional motor function will occur [16]. The specific finding in our cases we found that in 15 patients, were neurapraxia (first degree Sunderland) and in 3 patients were axontemesis (second degree Sunderland). To avoid unnecessary delay before indicated surgical reconstruction of the nerve is carried out, determining the degree of injury is important. Of equal importance is avoiding unnecessary exploration in the neurapraxia (first degree), axontemesis second degree which lead to more damage to the nerve and the regeneration process. This explain why we needed repeated EMGs and nerve conductions studies for better assessment and evaluation of the type of injury and the degree of functional recovery of the radial nerve. The incidence of fractures humerus per year in our trauma unit is about 100 cases per year with incidence of radial nerve palsy from 8 to 10 percent. The policy before in our department for the treatment of such cases of fractures humerus with radial nerve palsy was, exploration of the radial nerve with open reduction internal fixation for all the cases or external fixation for the open fractures cases but we found in all our explorations that there were only nerve contusions or neuropraxia without complete nerve cut except in one case with open fracture humerus which needed direct primay repair of the nerve with good return of function. Our results with 18 patients suffered from radial nerve palsy associated with humeral shaft fractures treated by closed interlocking intramedullary nail, as regarding the fractures union with early retrain of radial nerve and joint functions support the concept that, early exploration is not obligatory or an absolute indication in such type of fractures. There are many advantages of the closed interlocking nailing, as it work as internal splint which gives stability to the fracture for optimal position for radial nerve regeneration, it is less invasive and no need for radial nerve exploration which exposes the nerve to more trauma and ischemia due to violation of the nerve bed .We faced several limitations in our study as we did not have enough numbers of compound fractures cases to compare and also there were no cases with secondary nerve palsy after manipulation included in our study, so both groups were excluded from our study. The future research will included the cases with compound fractures and the cases with secondary nerve palsy after manipulation. Based on our study we found that, closed interlocking nailing for closed fractures humerus associated with radial nerve palsy, provides immediate stability and can be accomplished with a closed technique, brief operative time and minimum violation to the radial nerve, with resultant early return of optimal extremity function.

\section{References}

1. Hak DJ (2009) Radial nerve palsy associated with humeral shaft fractures Orthopedics 32: 111

2. Klenerman $L$ (1982) In: Wilson JN, editor. Watson-Jones fracture and join injuries. Edinburgh, London, Melbourne and New York: Churchill Livingstone Ch 21:578. ISBN: 044302825

3. Seddon HJ (1975) Surgical disorders of the peripheral nerves. Edinburgh: Churchill Livingstone; Ch 5:84. ISBN: 0-7817-1717-5.

4. Duthie BR, Bently G (1983) Mercer's orthopaedic surgery. (8 edns), London Edward Arnold.; Ch 12: 738-739. ISBN: 0-340-55163-1.

5. Mohler LR, Hanel DP (2006) Closed fractures complicated by peripheral nerve injury. J Am Acad Orthop Surg 14: 32-37.

6. Postacchini F, Morace GB (1988) Fractures of the humerus associated with paralysis of the radial nerve. Ital J Orthop Traumatol 14: 455-464.

7. Shao YC, Harwood P, Grotz MR, Limb D, Giannoudis PV (2005) Radial nerve palsy associated with fractures of the shaft of the humerus: a systematic review. J Bone Joint Surg Br 87: 1647-1652.

8. Lin J (2002) Locked nailing of spiral humeral fractures with or without radia nerve entrapment. Clin Orthop Relat Res: 213-220.

9. Pollock FH, Drake D, Bovill EG, Day L, Trafton PG (1981) Treatment of radia neuropathy associated with fractures of the humerus. J Bone Joint Surg Am 63: $239-243$

10. Sonneveld GJ, Patka P, van Mourik JC, Broere G (1987) Treatment of fractures of the shaft of the humerus accompanied by paralysis of the radial nerve. Injury 18: $404-406$

11. Böstman O, Bakalim G, Vainionpaa S, Wilppula $\mathrm{E}$, Patiala $\mathrm{H}$, et al. (1985) Immediate radial nerve palsy complicating fracture of the shaft of the humerus: when is early exploration justified? Injury 16: 499-502.

12. Böstman O, Bakalim G, Vainionpaa S, Wilppula E, Patiala H, et al. (1986) Radial palsy in shaft fracture of the humerus. Acta Orthop Scand 57: 316-319.

13. Ring D, Chin K, Jupiter JB (2004) Radial nerve palsy associated with highenergy humeral shaft fractures. J Hand Surg Am 29: 144-147.

14. Holstein A, Lewis GM (1963) Fractures of the humerus with radial nerve paralysis. J Bone Joint Surg Am 45: 1382-1388.

15. Shah JJ, Bhatti NA (1983) Radial nerve paralysis associated with fractures of the humerus. A review of 62 cases. Clin Orthop Relat Res: 171-176.

16. Jobe TM, Wright II EP (1998) Peripheral nerve injuries. In: S Terry Canale, etal editors. Campbell's operative orthopaedics, 9th ed. St. Louis: Mosby; Ch 81 3840-3841, ISBN 0-8151-2087-7. 